\title{
Anatomie artistique en prothèse faciale et muscles peauciers de la face et du cou ( $2^{\mathrm{e}}$ partie)
} Artistic anatomy in facial prosthesis and cutaneous muscles of the face and neck

\section{MOTS-CLEFS : \\ - Anatomie artistique, prothèse faciale, muscles peauciers de la face et du cou, platysma, fronto- occipitale, canons de proportion, règle de camper, anatomie topographique du visage humain, morphologie faciale, morphodifférenciation}

\section{KEYWORDS:}

- Artistic anatomy, facial prosthesis, cutaneous muscles of the face and neck, platysma, fronto-occipital, canons of proportion, camper's theory, topographical anatomy of the human face, facial morphology, morphodifferentiation

AOS 2015;271:37-48 DOI: $10.1051 / \mathrm{aos} / 2015106$ C) EDP Sciences 2015

\section{Résumé}

La première partie de ce cours d'anatomie donné à l'université Paris VI traite des muscles peauciers de la face et du cou et de leur importance dans le modelé du visage tant pour le chirurgien plasticien que pour le prothésiste facial. La deuxième partie traite de l'anatomie artistique. L'anatomie topographique ainsi que la morphologie des organes de la face et les canons esthétiques sont abordés.
Abstract

The first part of this course in anatomy given at the Universite Poris VI deals with the cutaneous muscles of the face and neck and their significance in face modelling for both plastic surgeons and facial prosthetists. The second part deals with the artistic anatomy. The topographical anatomy, organ morphology of the face and the aesthetic canons are also addressed.

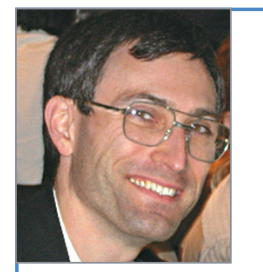

- Dr Christophe Bonnefoy, Lauréat de l'Académie Nationale de Chirurgie Dentaire. Enseignant au DU de Prothèse Faciale Appliquée. Université Paris VI

Dr Luc Chikhani, Responsable de l'unité de Stomatologie et de Chirurgie MaxilloFaciale, Chirurgien des hôpitaux, Expert en Stomatologie et Chirurgie Maxillo-Faciale. Hôpital Européen Georges Pompidou

Dr Jacques Dichamp, Responsable de l'enseignement du Diplôme. Universitaire de Prothèse Faciale Appliquée (1995 - 2010). Université Paris VI. Praticien hospitalier à l'Hôpital Européen Georges Pompidou et à la Pitié-Salpétrière. Université Paris VI. 


\section{RAPPELS}

La première partie de ce cours d'anatomie donné à l'université Paris VI, parue dans le numéro AOS 265 d'Octobre 2013, traitait des muscles peauciers de la face et du cou (MPFC) et de leur importance fondamentale dans le modelé du visage tant pour la prothèse faciale que pour la chirurgie plastique faciale.

Par définition, les MPFC $[1,2,4,7,9]$ :

\meuvent la peau et non des éléments du squelette facial ;

I appartiennent à la région superficielle de la face avec les muscles masticateurs masséters et temporaux;

I sorganisent en muscles péri-orificiels buccaux, narinaires et palpébraux ;

I dérivent tous du mésoderme para-axial du deuxième arc branchial ou arc hyoïdien (cartilage de Reichert);

I sont en conséquence tous innervés par le nerf facial (VII) ;

I provoquent des plis cutanés ou rides, toujours perpendiculaires à la direction de leurs fibres musculaires ou règle de Camper ;

I sont responsables de la fonction mimique impliquée dans les expressions et les émotions en agissant par synergie ou antagonisme ;
I interviennent, par une douzaine d'entre eux des plus superficiels, directement dans le modelé du visage nécessitant de facto une connaissance de type «mémoire de forme » permettant la construction de leur schématisation picturale et sculpturale;

I et ont in fine une plastique comparable à un drapé sur un personnage.

La classification actuelle, avec Christian Vacher et Jean-François Gaudy [4], considère les MPFC comme essentiellement annexés à l’oil, au nez et à la bouche. Le muscle annexé à l'orifice orbitaire est :

$\checkmark$ le muscle orbiculaire de l’eil avec 3 portions : palpébrale, orbitaire et lacrymale.

Les muscles annexés au nez et aux sourcils sont :

I le corrugateur, le procerus, le transverse, le dilatateur narinaire, labaisseur du septum nasal.

Les muscles annexés aux lèvres sont :

Ile muscle orbiculaire des lèvres parties interne et externe (cette dernière formée de tous les muscles peauciers qui s'insèrent sur l'angle oral);

$\checkmark$ les muscles incisifs ;

$\checkmark$ le muscle compresseur des lèvres ou muscle de la tétée;

Ile muscle mentonnier, mobilisateur indirect de la lèvre.

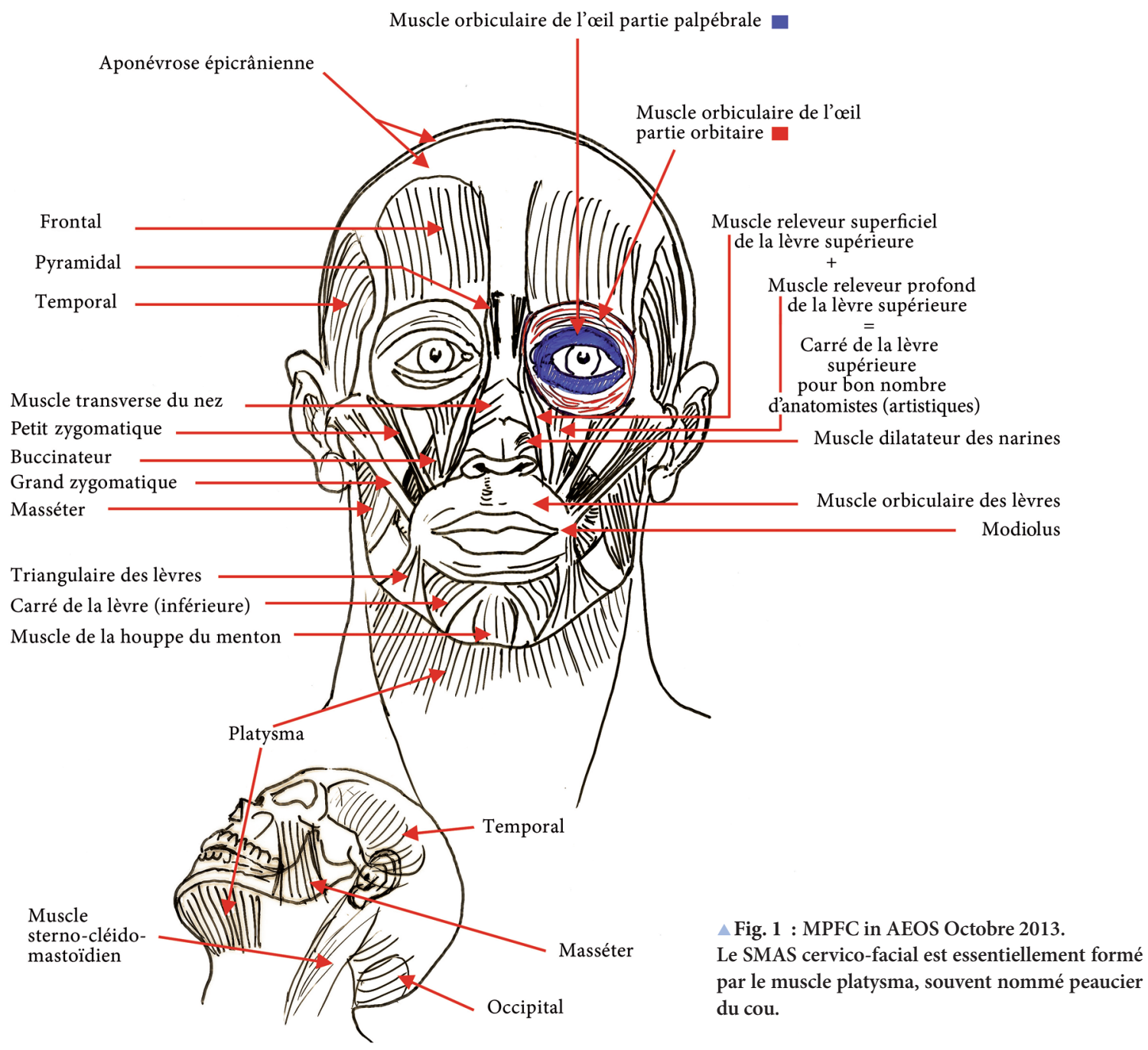




\section{ANATOMIE ARTISTIQUE EN PROTHĖSE FACIALE (PF)}

Les MPFC (objet du chapitre précédent), ne sont pas les seuls éléments à prendre en compte en anatomie pour le prothésiste ou le chirurgien plastique facial : le corps adipeux de la bouche, les muscles masticateurs et le système vasculaire de la face interviennent eux aussi de façon majeure dans le modelé d'un visage. Le corps adipeux de la bouche tend à faire disparaître le bord antérieur de la sangle massétérine et envoie son prolongement postérieur massétérique qui s'interpose entre les muscles masséter et buccinateurs. Chez les personnes ayant tendance à grossir, les repères osseux peuvent disparaître et provoquer, par exemple, un aspect « lunaire » du faciès chez les malades suivant une corticothérapie au long cours.

Lanatomie topographique du visage humain sera ensuite abordée car directement utile au prothésiste facial en passant en revue les régions à risque de décollement de la prothèse, les quatre organes majeurs de la face et les conséquences pratiques qui découlent de la connaissance de cette anatomie synthétique.

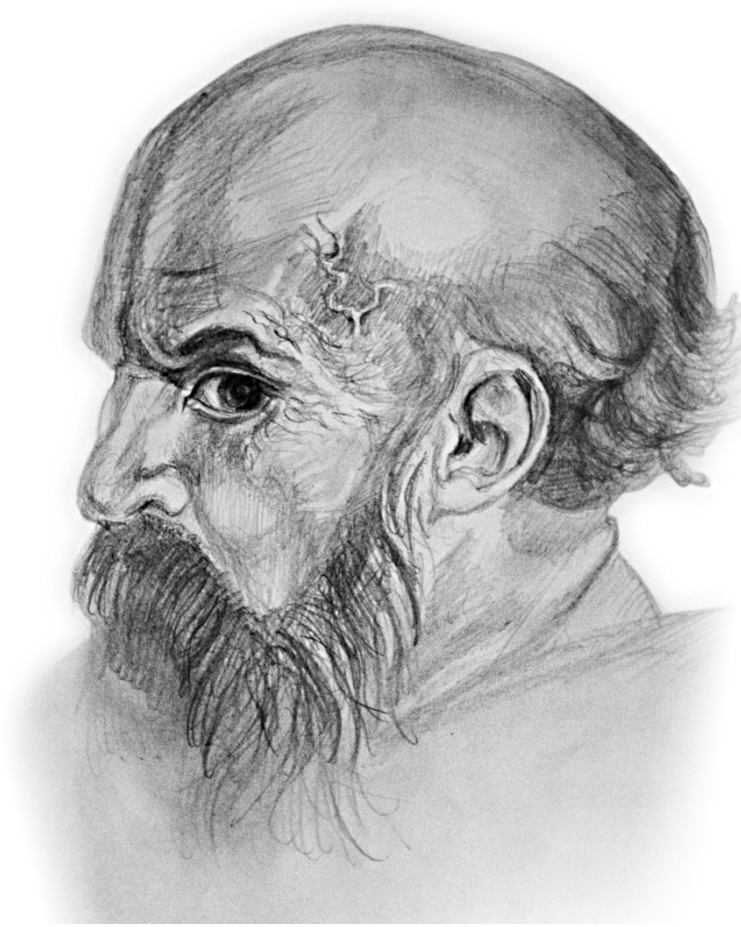

$\triangle$ Fig. 2 : dessin de l'auteur reproduisant la tête de Saint Pierre in « les quatre apôtres » d'Albrecht Dürer. (1471-1528). Un bel exemple artistique de la veine temporale superficielle.

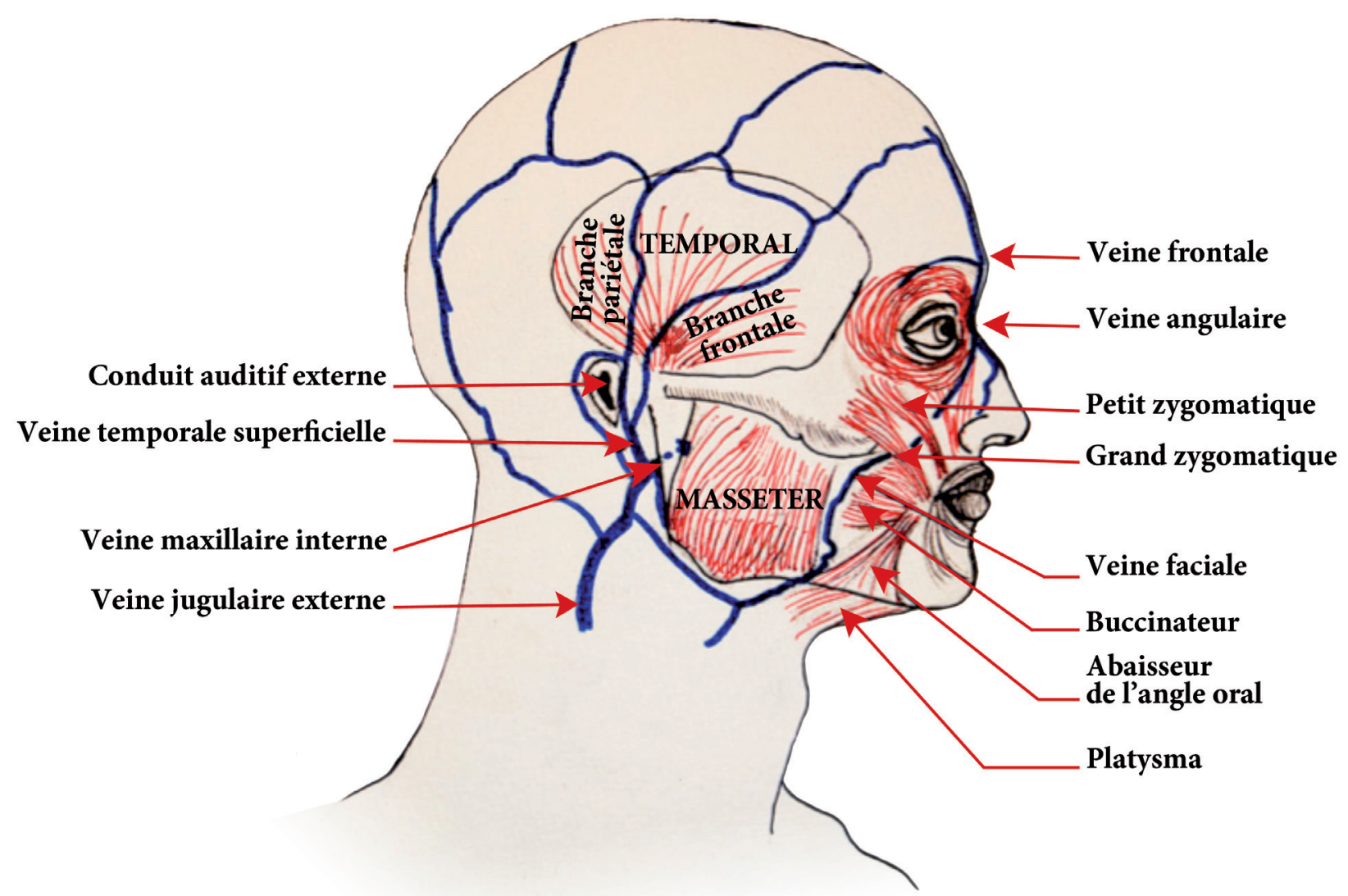

$\nabla$ Fig. 3 : dessin de l'auteur d'après Rouvière et Delmas représentant l'anatomie du système veineux de la face vue de profil. La veine faciale commence à l'angle interne de l'œil où elle porte le nom de veine angulaire qui s'anastomose avec la veine ophtalmique supérieure. La veine faciale se dirige vers le rebord basilaire mandibulaire en avant de la sangle massétérique, en dehors du buccinateur et en dedans des muscles petit et grand zygomatiques. La veine se termine inconstamment : dans la jugulaire interne ou le tronc thyro-linguo-facial. C'est une veine importante en anatomie artistique du visage avec la veine temporale superficielle. Elle est toujours située en dehors de l'artère et en arrière dans son trajet jugal jusqu'à l'angle antéro-inférieur du masséter. 
Régions de la voute cranienne

1. région frontale

2. région pariétale

3. région temporale

4. région sous-temporale (infra-temporale)

5. région occipitale

\section{Régions de la face}

6. région nasale

7. région labiale / buccale

8. région mentonnière

9. région orbitaire

10. région sous-orbitaire (infra-orbitaire)

11. région génienne

12. région zygomatique

13. région parotido-massétérine

\section{Régions du cou}

(14-15-18-19-20)

14. région muchale

15. région sterno-cléido-mastoïdienne

18 région sus-hyoïdienne-médiane

19. région de la loge sous-mandibulaire

20. région parotidienne
Régions primordiales en anatomie artistique du visage :

- région orbitaire

- région labiale

- région nasale

- région mentonnière

- région zygomatique

- Pavillon de l'oreille

Ponts antropométriques de repérage en anatomie artistique du visage (reconstruction volumique globale)

1. nasion Forme $+/$ - losangique
2. zygion

3. bregma

4. gnathion

5. gonion

15

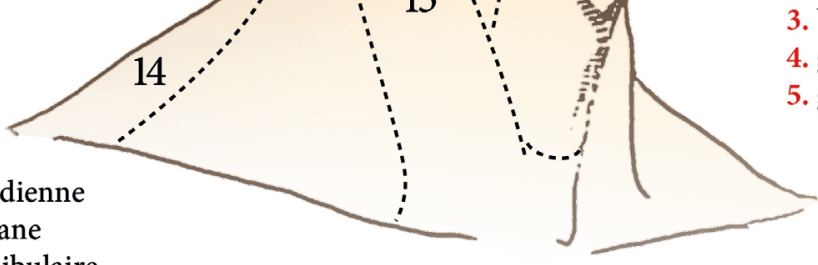

Régions de la tête et du cou en vue latérale

$\triangle$ Fig. 4 : dessin de l'auteur représentant les régions de la tête et du cou en vue latérale. La connaissance des repères topographiques est essentielle lors d'une reconstruction chirurgicale ou prothétique parce qu'ils sont déterminants dans l'appréciation du modelé du visage. La région zygomatique constitue ainsi une région charnière lors des pertes de substance importantes. Quelques points anthropométriques principaux de repérage du visage au niveau cutané correspondent au point gnathion, point bregma, point zygion $\mathrm{D}$ et $\mathrm{G}$, point nasion et point gonion (ce dernier étant utile pour sculpter ou peindre un visage de profil).

En outre, la schématisation du visage humain est basée sur la construction de la tête en anatomie artistique. Toutes ces considérations déboucheront sur :

$\checkmark$ le problème de la morphologie faciale qui occupe les artistes depuis l'antiquité ;

I'approche moderne du canon qui repose sur la statistique ;

$\checkmark$ les canons artistiques du visage qui obéissent à un système de proportions qui détermine la morphologie faciale idéale ;

$\checkmark$ la morphodifférenciation en fonction du sexe et de lâge qui rejoint également le système des canons morphologiques avec des effets de modes auxquels il est difficile d'échapper quelles que soient les époques.

\section{Système vasculaire de la face : artères, veines et expressions du visage}

Seules les veines superficielles nous intéressent dans la plastique faciale. Il s'agit des veines frontales, angulaires, faciales et temporales $[3,9]$.
Elles sont utilisées en prothèse faciale comme artifices de vieillissement du visage. Elles sont davantage visibles chez le sujet âgé, chauve ou lors des efforts violents.

\section{Anatomie topographique du visage humain}

Cette anatomie est plus particulièrement nécessaire en chirurgie et en prothèse selon l'importance de la région concernée par une reconstruction chirurgicale et/ou prothétique $[2,5,9,12]$.

\section{Schématisation des régions (fig. 4).}

Il est impératif, tant pour le prothésiste que pour le chirurgien, de connaître les régions à risque de décollement de la prothèse $[3,12]$. Il s'agit de :

$\checkmark$ La région orbitaire;

$\checkmark$ La région labiale;

$\checkmark$ La région génienne ;

I La région nasale. 


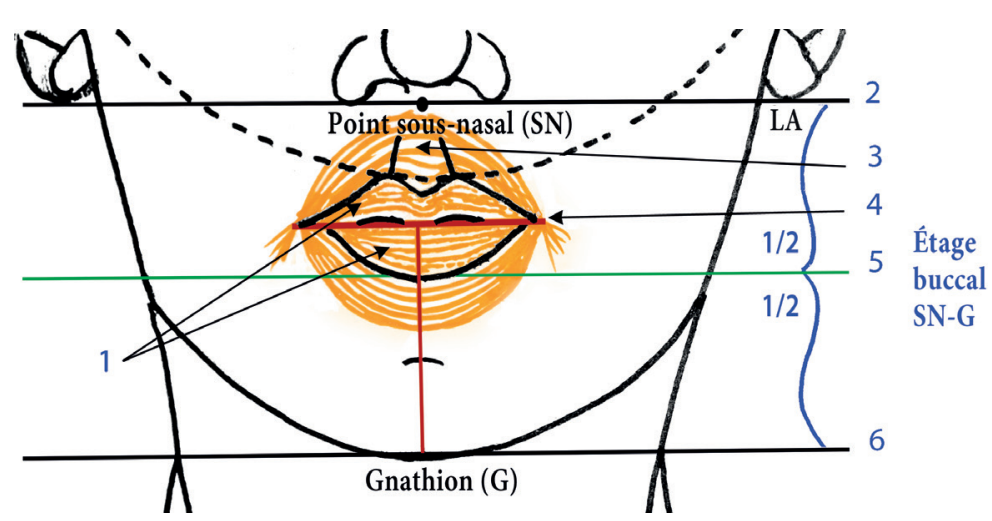

$\triangle$ Fig. 5 : dessin de l'auteur représentant l’orbiculaire des lèvres dans létage buccal, en orange, compris entre le point sous-nasal (SN) et le point gnathion $(\mathrm{G})$. En rouge, le « $\mathrm{T}$ » de Léonard de Vinci : la largeur de la ligne bicommissurale correspond à la distance séparant le milieu de celle-ci au point gnathion sur l'axe vertical de symétrie. 1 : Vermillon de la lèvre supérieure et inférieure. 2 : Ligne horizontale SN- Bord inférieur du lobe auriculaire (LA) correspondant à la base du nez ou limite supérieure de létage facial inférieur. 3 : gouttière nasale ou philtrum. 4 : commissure encore appellée modiolus ou angle oral. 5 : en vert, la ligne médiane de l'étage buccal passant par le bord inférieur de la lèvre inférieure. 6 : ligne horizontale passant par le bord inférieur de la symphyse mentonière ou point gnathion $(G)$ ou limite inférieure de létage facial inférieur.

\section{Les quatre organes}

\section{La bouche $[4,7]$}

Par définition, la bouche constitue un sphincter oral à fonction mimique intégrée.

Sa forme est déterminée par la courbure des lèvres avec amincissement aux commissures, appelées encore angle oral ou modiolus. La lèvre supérieure est plus large et plus proéminente que la lèvre inférieure selon le canon. Les deux lèvres de par leurs courbures respectives forment de face un "canapé " avec plicature centrale correspondant à la gouttière nasale ou philtrum. Pour Léonard de Vinci, la largeur des lèvres au niveau de la ligne bicommissurale est à peu près égale sur le visage de face à la distance qui sépare cette dernière du gnathion sur la ligne médiane (13).

\section{L'oreille}

Par définition, il s’agit d'un pavillon en forme de point d'interrogation.

Le repérage du pavillon de loreille se fait aisément, il est ainsi parallèle à la ligne du dos du nez dans létage moyen de la face sur un visage vu de profil. Il permet de reconnaitre la ligne ou plan de Camper par la distance méat acoustique externe (matérialisé au niveau cutané par le tragus) - base du nez (matérialisée au niveau cutané par le bord inférieure de l'aile du nez). Le pavillon de loreille possède une grande variété de formes individuelles (se référer à loreille controlatérale si elle existe).

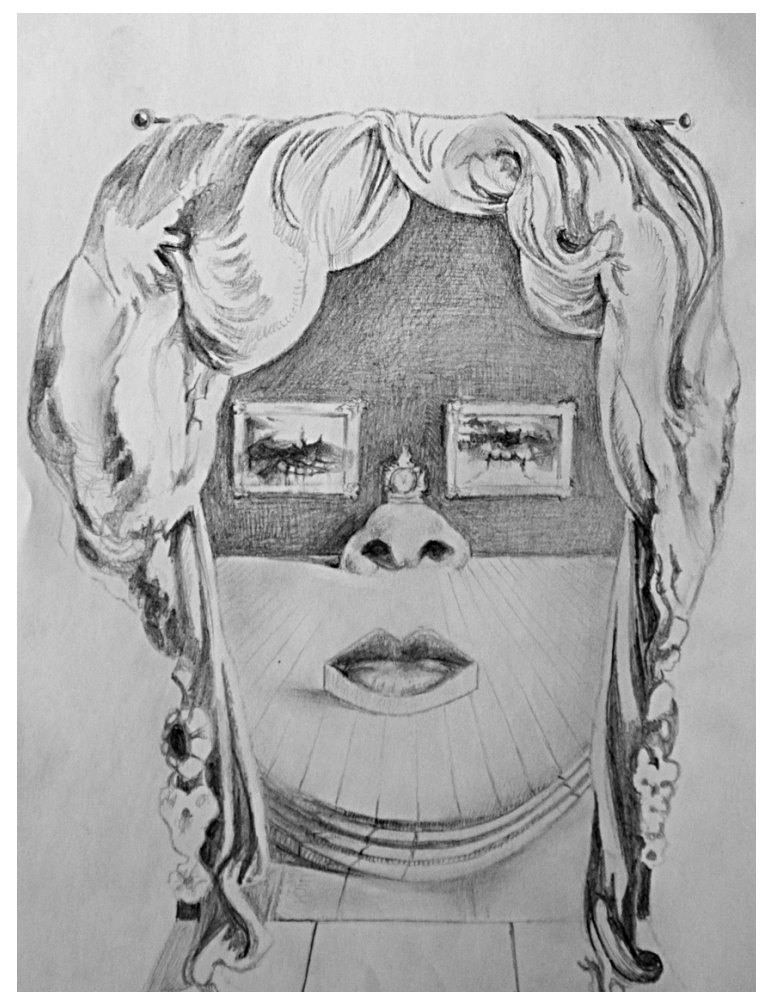

$\triangle$ Fig. 6 : dessin de l'auteur représentant le visage de Mae West avec les lèvres en canapé d'après la toile de Salvador Dali.

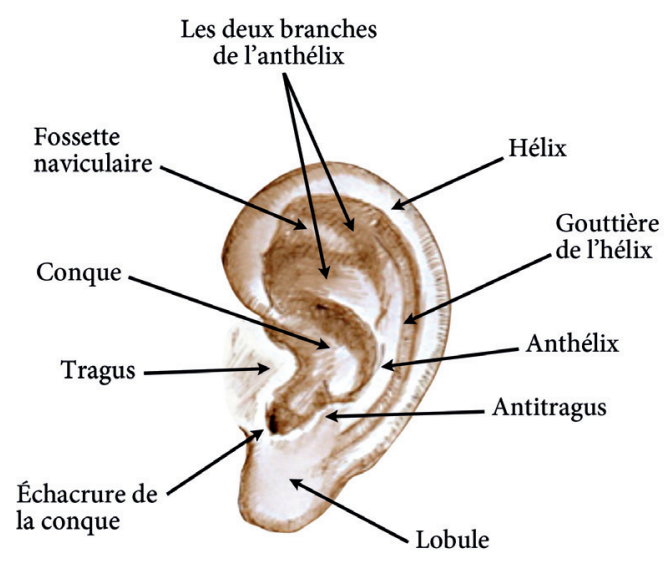

$\triangle$ Fig. 7 : dessin de l'auteur représentant l'anatomie du pavillon de l'oreille

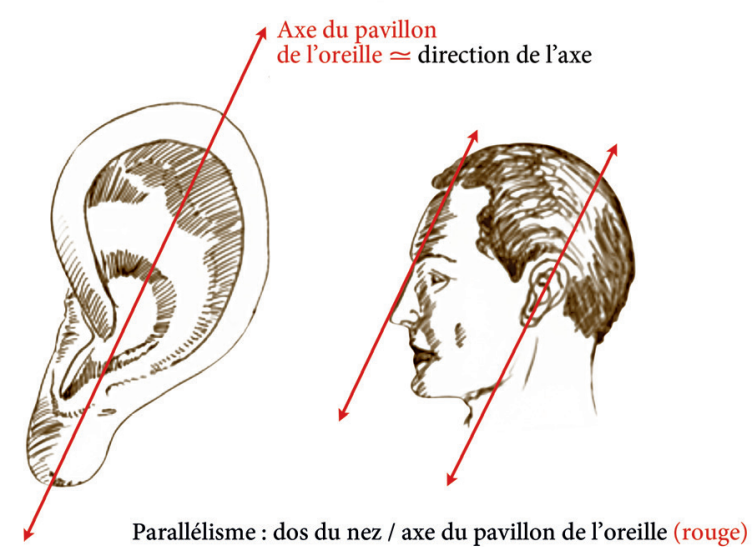

$\triangle$ Fig. 8 : dessin de l'auteur montrant la position de l'oreille dans létage moyen de la face vue de profil. 


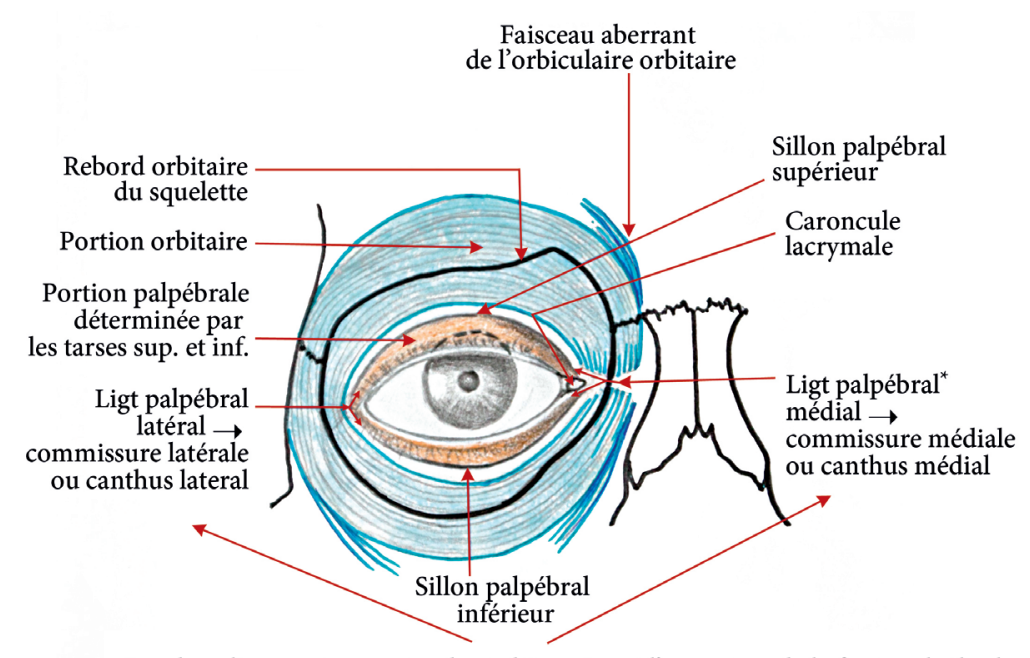

Les deux ligaments en se tendant, déterminent l'ouverture de la fente palpébrale en s'attachant au rebord orbitaire du squelette. L'œil de face, le sujet regardant devant lui, présente un iris amputé d'1/4 de sa hauteur environ par la paupière supérieure.

* encore appelé tendon direct du muscle orbiculaire de l'œil.

$\triangle$ Fig. 9 : dessin de l'auteur représentant l'anatomie de lorbiculaire orbitaire tributaire de l'aspect « tombant » du côté latéral du rebord orbitaire du squelette.

$\nabla$ Fig. 10 : dessin de lauteur de la pyramide nasale et et du buste de Nefertiti provenant de l' atelier de Thoutmosis, mais dont la modernité et l'exceptionnel état de conservation étonne toujours au point que cette œuvre est considérée par certains auteurs avec suspicion quant à son authenticité. Toutefois les analyses les plus sophistiquées n’ont jamais pu prouver qu'il s'agissait d'un faux mais d'un noyau calcaire enrobé de platre corrigeant l'arête nasale, les pommettes, les joues et le sillon nasogénien jusqu'à la commissure, constituant le premier lifting connu de l'histoire.

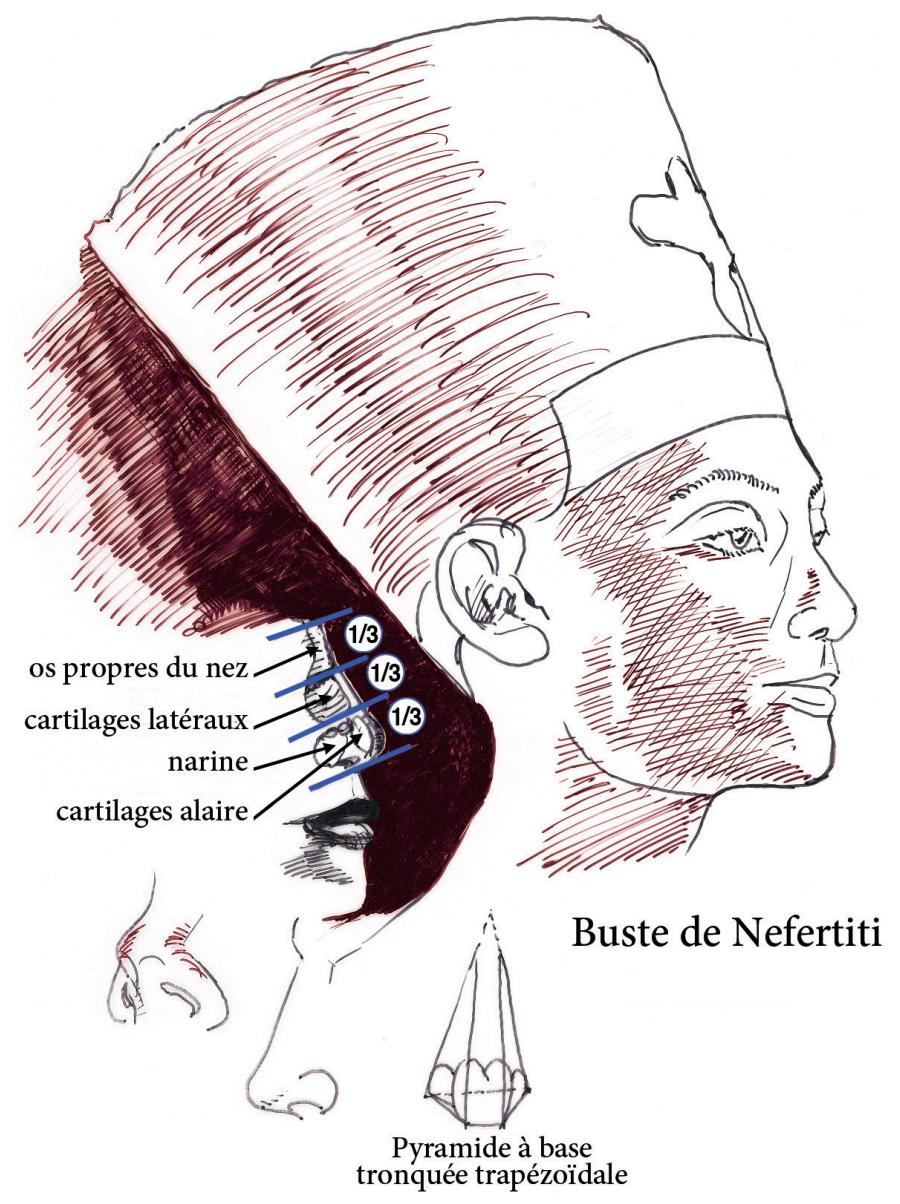

\section{L'œil}

Le globe oculaire est masqué en haut et en bas par, respectivement, les paupières supérieure et inférieure correspondant respectivement aux tarses supérieur et inférieur et légèrement déplacé vers la portion nasale de lorbite. Les paupières déterminent la forme en amande des yeux et se présentent sous la forme de coquilles.

Il est traditionnel de représenter l'angle interne de l'oeil ou canthus médial plus bas que l'angle externe ou canthus latéral mais en fait, il faut toujours copier l'oeil symétrique résiduel. Le repérage de la pupille est important (plan bipupillaire), celle-ci correspondant à louverture centrale du diaphragme vertical et circulaire qu'est l'iris, ce dernier étant seul observable puisque la cornée qui le recouvre est transparente.

Le bulbe oculaire peut être considéré comme un renflement distal, en architecture multicouche, du nerf optique.

\section{Le nez}

Par définition, le nez est une pyramide tronquée à base trapézoïdale sélargissant de la racine à la base dont la largeur de cette dernière est de $3,89 \mathrm{~cm}$ dans la population masculine et de $3,66 \mathrm{~cm}$ dans la population féminine. La partie inférieure cartilagineuse narinaire forme de face un bourrelet ovoïde qui peut être pris comme module de la hauteur totale du nez et équivaut au tiers de celle-ci.

La formule des trois tiers donne le module de proportion de la pyramide nasale :

I un tiers supérieur correspond aux os propres du nez. I un tiers moyen correspond aux cartilages latéraux I un tiers inférieur correspond aux cartilages alaires. Les proportions nasales obéissent de profil au triangle rectangle 3-4-5 correspondants respectivement (fig. 11) : I à la largeur du bord postérieur de laile à la pointe du nez,

Ià la hauteur du bord postérieur de l'aile au nasion cutané,

I au dos du nez du nasion cutané à la pointe.

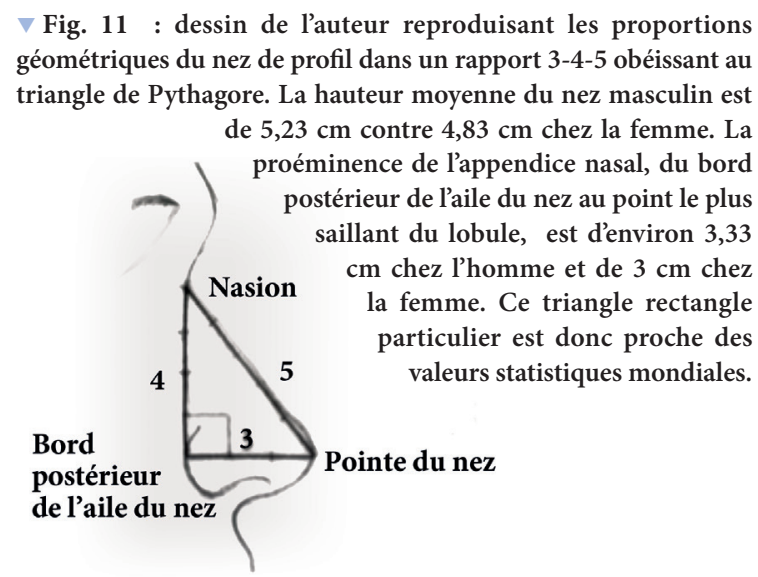




\section{Conséquences pratiques en prothèse} plastique faciale

La connaissance de l'anatomie est non seulement indispensable en chirurgie mais permet encore de tirer des conséquences pratiques en prothèse plastique faciale [3, 12]. Ainsi, il convient :

1. de retrouver les points anthropométriques de repérage et le côté symétrique pour les organes pairs en respectant l'axe de symétrie et pour cela, si possible, réaliser une empreinte la plus complète possible, notamment la restitution des trois étages de la face, car elle constitue la base du travail ;

2. de prendre de préférence des moulages et des photographies préopératoires, indispensables lors de la reconstruction ad integrum de la pyramide nasale par exemple, afin dêtre le plus fidèle possible en évitant la confrontation des points de vues esthétiques du patient et du prothésiste ;

3. d'éviter la reconstitution des régions labiales et géniennes puisqu'elles constituent des centres musculaires peauciers. Or ces muscles exercent des contraintes mécaniques beaucoup plus importantes quoon ne l'ima- gine au vu de leur minceur chez lécorché. En effet, ils exercent des forces centrifuges par rapport à la pièce prothétique mais non orientées dans le même sens, ce qui crée des composantes tangentielles par rapport à la direction du vecteur force principal représentant la traction musculaire des peauciers concernés. Or ce sont ces composantes qui sont les plus nocives pour les adhésifs utilisés en prothèse plastique faciale qui, comme toutes les colles, résistent mal aux forces de cisaillement. On peut se retrouver ainsi devant une aporie prothétique qui plaide donc en faveur de la reconstruction chirurgicale.

4. de contrôler l'exérèse chirurgicale par des moulages préopératoires permettant non seulement la résolution des aspects esthétiques (cf. point 2 ) mais encore de contrôler et de simuler l'impact de cette exérèse chirurgicale. Il est donc nécessaire de tenir compte des régions à haut risque de décollement de la prothèse où toute incursion dans une région donnée équivaut souvent à l'impotence fonctionnelle totale de celle-ci. On privilégiera, comme en Implantologie par exemple, l'importance d'une concertation préopératoire chirurgien-prothésiste.

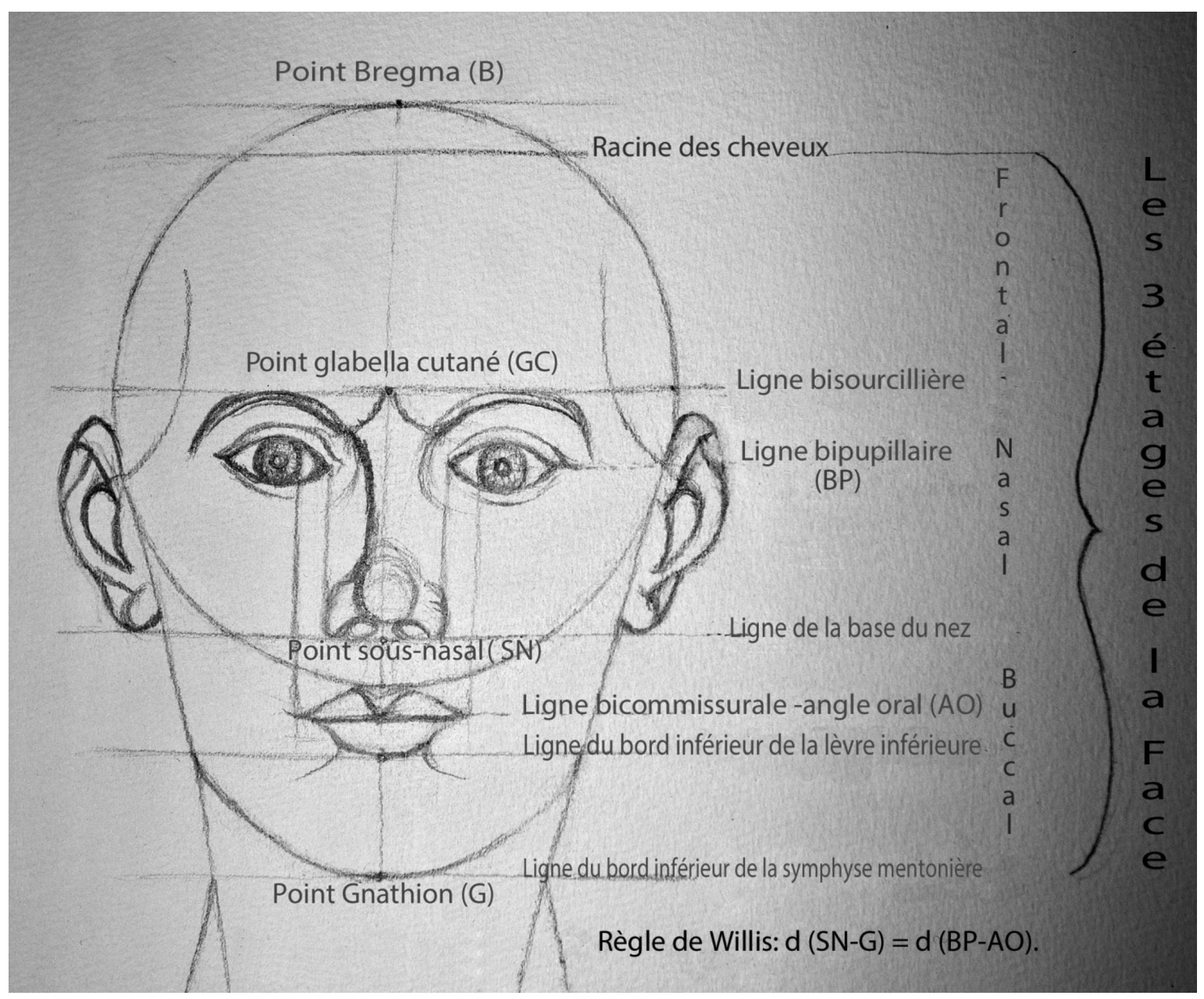

$\triangle$ Fig. 12 : dessin de l'auteur montrant la construction du visage de face, l'homme étant censé regarder devant lui, selon le canon statistique de Fristch qui comporte une part subjective : la distance bregma-racine des cheveux dont les dimensions ne sont pas fixées dans le sens vertical. Toutefois, avec ce canon artistique, un débutant peut peindre et sculpter un visage humain vu de face. 


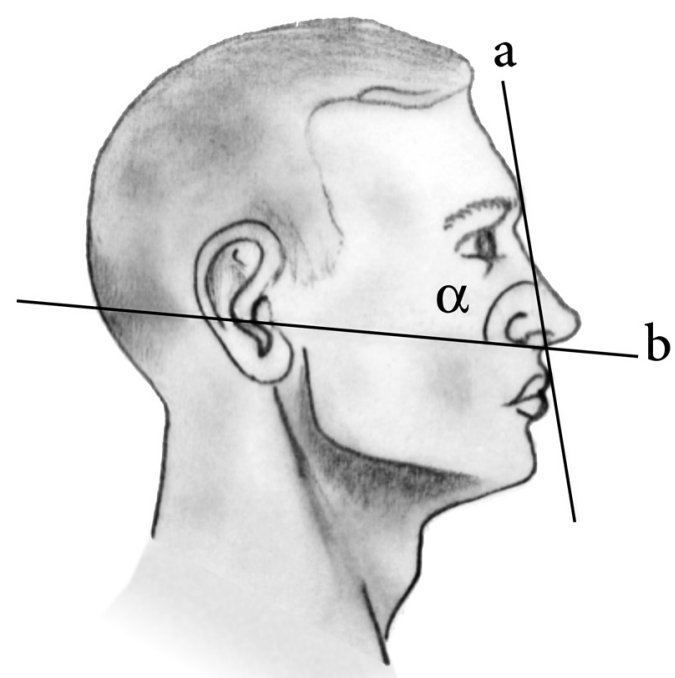

$\triangle$ Fig. 13 : dessin de l'auteur matérialisant l'angle facial a de Camper sur le vivant. Les rapports d'étendue ont été exagérés par les sculpteurs anciens dans les têtes de dieux ou de héros afin d'idéaliser le profil de la face humaine en augmentant l'ampleur du front. En conséquence le frontal se retrouve dans le prolongement du dos du nez, langle atteignant $90^{\circ}$. Cet angle, utilisé en paléoanthropologie, a été considéré comme matérialisant le développement du tube neural à la partie frontale de son extrémité céphalique, soit au niveau des lobes frontaux. Or ces derniers interagissent avec d'autres aires cérébrales dans la planification, le langage et le mouvement volontaire. Les grecs semblent bien en avoir fait l'attribut principal du surhomme, suivi par des théoriciens racistes jusqu'au vingtième siècle, ignorant que le système nerveux central forme un ensemble interconnecté et indissociable à la manière d'une équipe sportive et que l'intelligence n'est pas une donnée anthropométrique. En outre, il ne constitue qu'un indicateur de lévolution dans l'espèce humaine et ne tient pas compte de la base du crâne par exemple. Camper signalait déjà dès 1791 que « la comparaison de l'angle facial sétablit sans référence à la masse cérébrale ». Or cette dernière était considérée à lépoque de manière erronée comme une mesure de l'intelligence et même plus tard encore au cours du XIX ${ }^{e}$ siècle. Peut-on considérer réellement Camper comme le père du racisme scientifique ? A son insu, certainement.

\section{Schématisation artistique du visage humain}

\section{Construction actuelle de la tête en anatomie artistique}

La construction schématique de la tête selon le canon de FRISTCH (fig. 12) se fait à l'aide de la distance Bregma-Gnathion, de la hauteur de la face divisée en trois étages dégale hauteur en lui amputant la distance Bregma-racine des cheveux, d'un cercle qui rend compte de laspect de la calotte crânienne de face et qui a pour centre le point Glabella cutané, le milieu ou point le plus proéminent de la ligne bisourcilière (ou biophryaque), et dont le diamètre vertical va du point bregma au bord inférieur de la lèvre inférieure qui se trouve en plein milieu de l'étage inférieur ou buccal de la face. La largeur de la base du nez est égale à la distance séparant les angles internes des yeux ou distance intercanthale (environ $33 \mathrm{~mm}$ en moyenne), et enfin la règle de Willis fixe la place de la ligne bipu- pillaire. Cette dernière est divisée en 5 parties égales ayant comme unité la distance canthus interne-canthus externe correspondant à la largeur du cercle à ce niveau (Vinci, Dürer). Ce qui implique schématiquement que la largeur de la base du nez ou distance intercanthale est environ égale à la distance canthus interne-canthus externe, c'est-à-dire à la largeur des yeux. Les oreilles, rappelons-le, sont toute entières contenues dans l'étage moyen ou nasal. Ceci ne constitue en clinique qu'une base de travail approximative qui ne tient aucunement compte des variabilités morphologiques individuelles ou ethniques et en particulier de l'aspect brachycéphale ou dolichocéphale du patient étudié.

\section{Angle facial et ethnie}

L’angle facial de Camper (fig. 13) est l'angle $\alpha$ déterminé de profil au niveau cutané par deux droites qui se croisent et dont l'une (a) passe par la partie la plus saillante du front (la glabella cutanée) et la lèvre supérieure et l'autre (b) par le tragus et la base de la pyramide nasale. Au niveau squelettique, cet angle sert de repère anthropométrique en paléoanthropologie:

I a : glabella- partie vestibulaire du bord incisif des incisives maxillaires correspondant approximativement au niveau cutané à la Ligne glabella cutanée - lèvre supérieure ;

I b : ligne méat acoustique externe- épine nasale matérialisant le plan de Camper correspondant approximativement au niveau cutané à la ligne tragus - base du nez;

$1 \alpha$ : angle facial de Camper.

Il ne faut pas confondre l'angle facial de Camper avec la règle de Camper qui explique laction des muscles peauciers. Langle facial affiche les valeurs moyennes suivantes :

$180^{\circ}$ à $82^{\circ}$ dans l’ethnie caucasienne ;

$175^{\circ}$ dans lethnie asiatique ;

$170^{\circ}$ dans l'ethnie noire ;

$195^{\circ}$ pour les bustes romains ;

$\ 100^{\circ}$ pour les bustes grecs comme l'Apollon pythique.

\section{Construction de la tête en trois dimensions}

Elle se caractérise par l'introduction de la perspective dans le canon de Fristch.

La tête devient ainsi un solide imaginaire dont les subdivisions permettent de placer tous les éléments anatomiques. Les cercles deviennent ainsi des boules et les lignes, des ellipses. Les mesures anthropométriques constituent une base de travail de façon à aller plus rapidement à l'essentiel pour un patient donné. Le modelé du visage en « $3 \mathrm{D}$ " doit toujours être étudié sous plusieurs angles tout en privilégiant la vue frontale comme le faisaient les sculpteurs de la renaissance tel Michel-Ange qui travaillait les blocs 


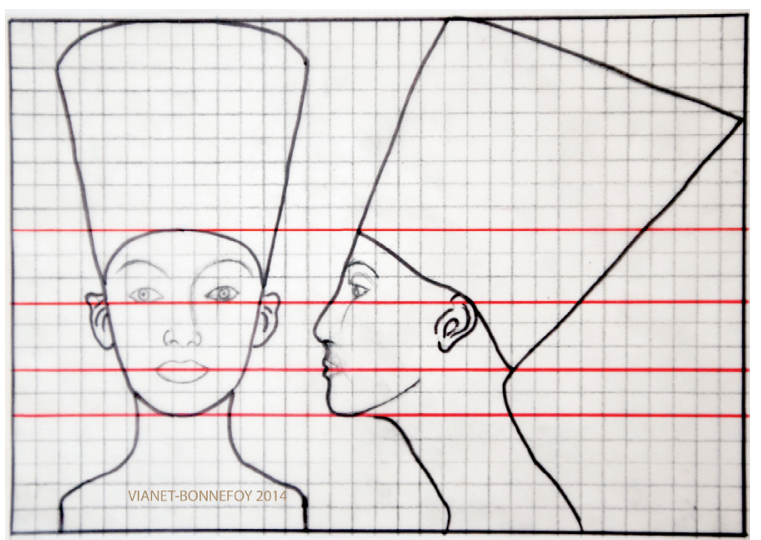

$\Delta$ Fig. 14 : dessin de l'auteur représentant la tête de Nefertiti d'après des études égyptologiques. Elle obéit à un système métrique particulier qui est retrouvé dans le canon amarnien puisque la largeur du doigt $(1,85 \mathrm{~cm})$ en constitue l'étalon. En conséquence, elle serait un modèle d'atelier servant de base à la représentation et à la reproduction de la tête de la souveraine. 4 lignes horizontales sont remarquables : menton, bicommissurale, intercanthale, bord inférieur de la coiffe. Notez que la ligne ophryaque n'est pas clairement définie par ce canon mais que la ligne verticale bord interne pupillaire-commissure est présente.

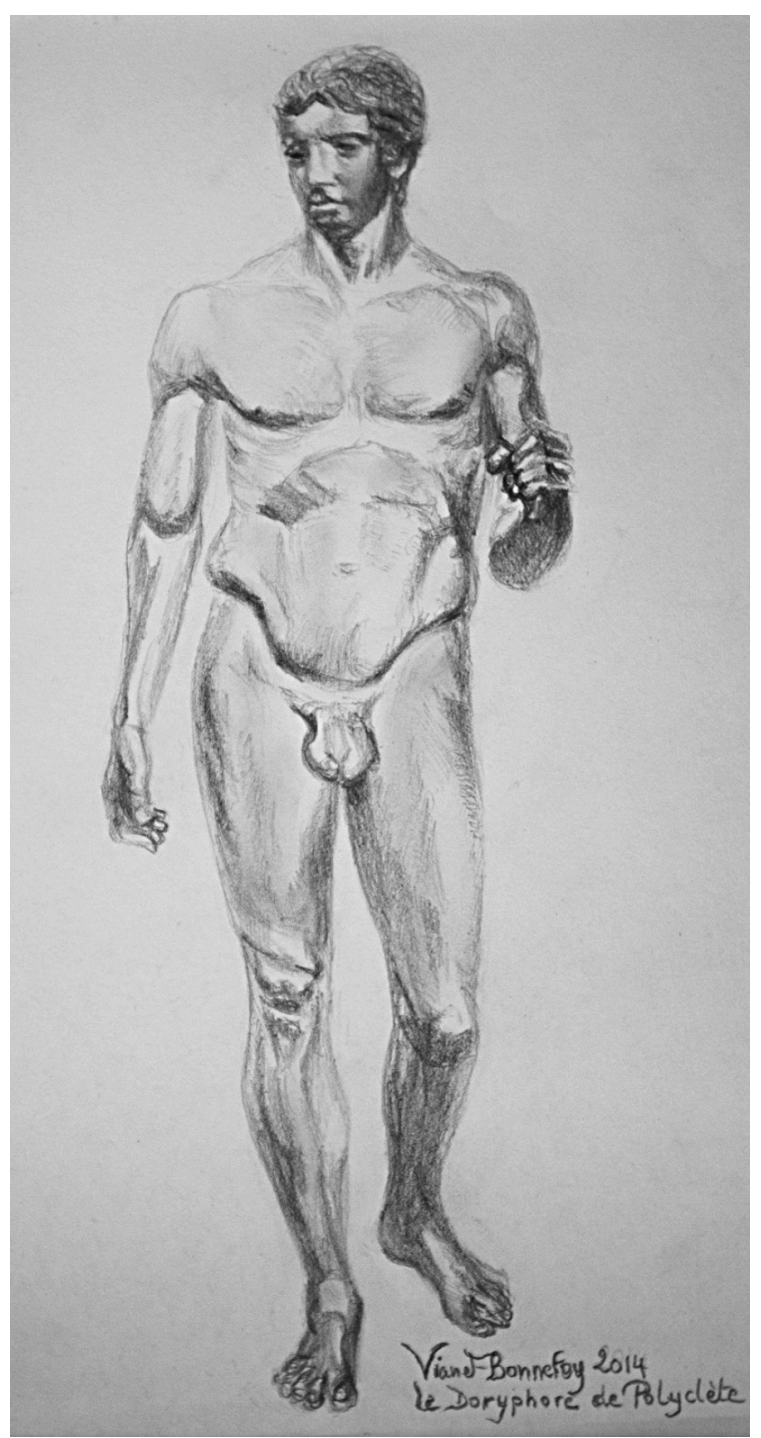

$\triangle$ Fig. 15 : dessin de l'auteur représentant la statue du Doryphore de Polyclète ou canon 7 1/2. de marbre en technique soustractive par balayage à la manière d'un scanner-imprimante $3 \mathrm{D}$.

\section{Canons : le problème de la détermination de la morphologie idéale}

\section{Canons de proportionnalité}

La schématisation du visage humain nous amène naturellement à nous poser la question de l'utilité de posséder un canon de proportionnalité facilitant la reproduction $[6,8,13]$.

Le mot " canon " signifie littéralement " règle " ou «loi formelle». De nombreuses tentatives ont eu cours à toutes les époques afin d'enfermer la diversité des formes du corps humain dans des rapports géométriques.

\section{Le canon égyptien}

Il existe en fait trois canons recensés au cours des différentes périodes pharaoniques dont le plus connu est le deuxième qui divise le pied en trois parties égales, aboutissant à une hauteur de l'individu fixée arbitrairement à dix-neuf modules. Selon ce canon, la tête est représentée par deux modules et deux tiers.

\section{Le canon de Polyclète}

La statue du Doryphore de Polyclète a conduit à l'adoption du "canon de Polyclète ». La hauteur du corps est divisée en sept modules et demi dont l'unité est la tête. Ce canon convient à un travailleur de force, un champion de poids et haltères, il s'en dégage une impression de puissance.

\section{Le canon de la renaissance}

Le canon de Vitruve est représenté ici par Léonard de Vinci dans son Codex. Il s'agit en fait du canon de Lysippe, le plus usité dans l’antiquité grecque. Le corps humain est contenu dans un cercle inscrit dans un carré dont le nombril est le centre. La hauteur du carré est divisée en huit parties égales dont la tête, soit la distance gnathion-bregma, est l'unité.

Il définit un type plus longiligne que le canon de Polyclète et correspond davantage à notre conception $\mathrm{du}$ « mannequin » actuelle prônée dès le début du vingtième siècle par Richer [8].

\section{Le canon de Fritsch}

Le canon de Fritsch ou canon 73/4 est un canon moderne basé sur la statistique d'un type intermédiaire aux deux précédents. Il présente des caractéristiques de subdivisions en modules permettant la reconstitution du visage bien que celui-ci se présente de façon fragmentaire. C’est celui que nous avons utilisé plus haut pour la construction du visage (fig. 12). 


\section{Statistiques et homme idéal}

On a beaucoup critiqué la tentative de Paul Richer [8] d'établir un prototype d'homme moyen conventionnel dont les caractéristiques morphologiques sont données par son écorché. C’est tout le problème de l'homme idéal, sorte de modèle, de mannequin censé représenter la beauté offerte à l'éducation des jeunes artistes. Pourtant, il suffit d'ouvrir un tant soi peu un manuel scolaire de philosophie pour s'apercevoir que la beauté a une valeur purement subjective. Kant ne dit-il- pas que " quand quelqu'un dit d'une chose qu'elle est belle, il attribue aux autres la même satisfaction » ? C'est cette espèce de totalitarisme artistique que l'on reproche à Richer et à bien d'autres. En définitive, il nous faut faire attention au fantasme totalitaire artistique ou scientifique du canon statistique!

Différentes approches existent :

$\checkmark$ l'approche statistique théorique avec une morphologie basée sur la moyenne des caractéristiques anatomiques de la morphologie cranio-faciale;

$\checkmark$ l'approche au cas par cas pratique et clinique ;

$\checkmark$ l'approche géométrico-symbolique avec certains artistes comme Dali et confrères tels Ricketts (cité in 13) qui introduisent le fameux nombre d'or ou rapport $\Phi$, dit de » divine proportion » équivalent à $(\mathrm{a}+\mathrm{b}) / \mathrm{a}=\mathrm{a} / \mathrm{b})$, impliquant que a vaut $(1+\sqrt{ } 5) / 2 \mathrm{si}$ b est l'unité soit environ 1,618, dans les proportions du visage.

«Au nom de la morphologie humaine, on établit un canon dont on parle comme d'un attracteur (mathématique), un centre autour duquel gravite toutes les

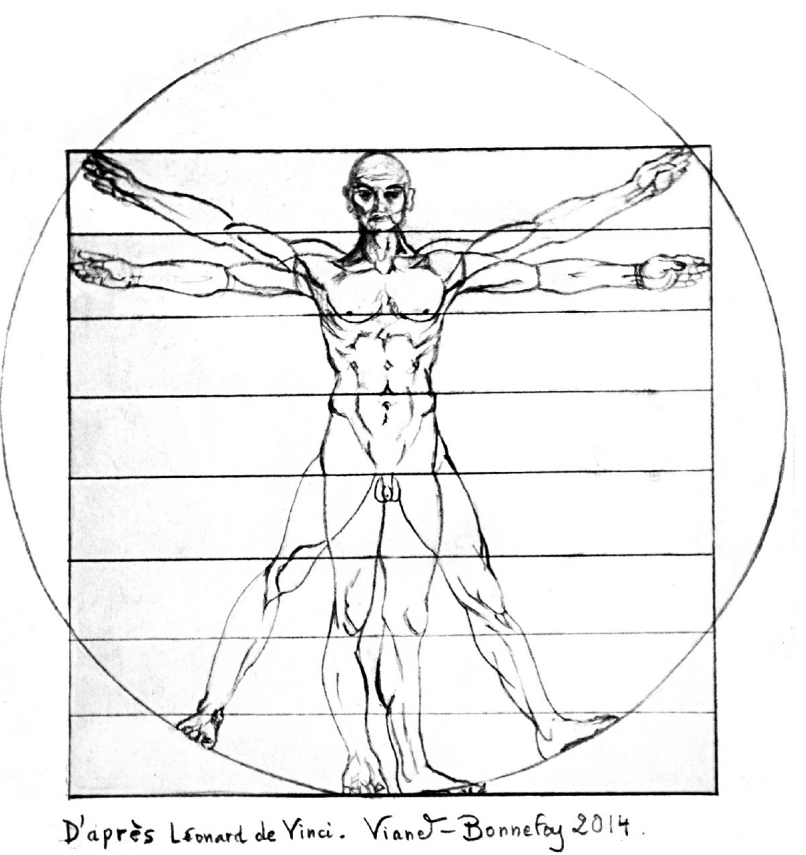

$\triangle$ Fig. 16 : dessin de l'auteur reprenant le Canon de Vitruve reproduit par Léonard de Vinci ou canon 8. individualités, qui n'en représente aucune, mais qui se rapproche de toutes à la fois! » (Laubry in 6). Enfin, le canon en tant que modèle imposé par les medias et la mode ne s'apparente-t-il pas à un racisme, ou en tout cas à un système discriminatoire ? Cette question reste ouverte et dépasse naturellement par ses prolongements philosophiques, le cadre de cet article.

\section{MORPHODIFFÉRENCIATION EN FONCTION DU SEXE ET DE L'ÂGE}

\section{L’homme idéal}

Le « David » de Michel Ange constitue un bel exemple d'aristocratie du visage

Il obéit au canon de la renaissance caractérisé par son profil grec et a contribué à évoquer le paradigme de l'idéal masculin.

\section{La femme idéale}

Un exemple des canons de l'aristocratie du visage est ici représenté avec la toile de Gabrielle d'Estrées peinte par François Clouet vers 1570. Le tableau joue du contraste entre la laide nourrice fonctionnelle et la belle dame idéalisée avec le visage ovalaire, les traits fins, le front haut (canon de la beauté au moyen-âge jusqu’à la renaissance) et la pyramide nasale longue. Certains auteurs comme Ricketts (cité in 13) vont jusqu’à introduire à la suite de Léonard de Vinci le fameux nombre d'or dans

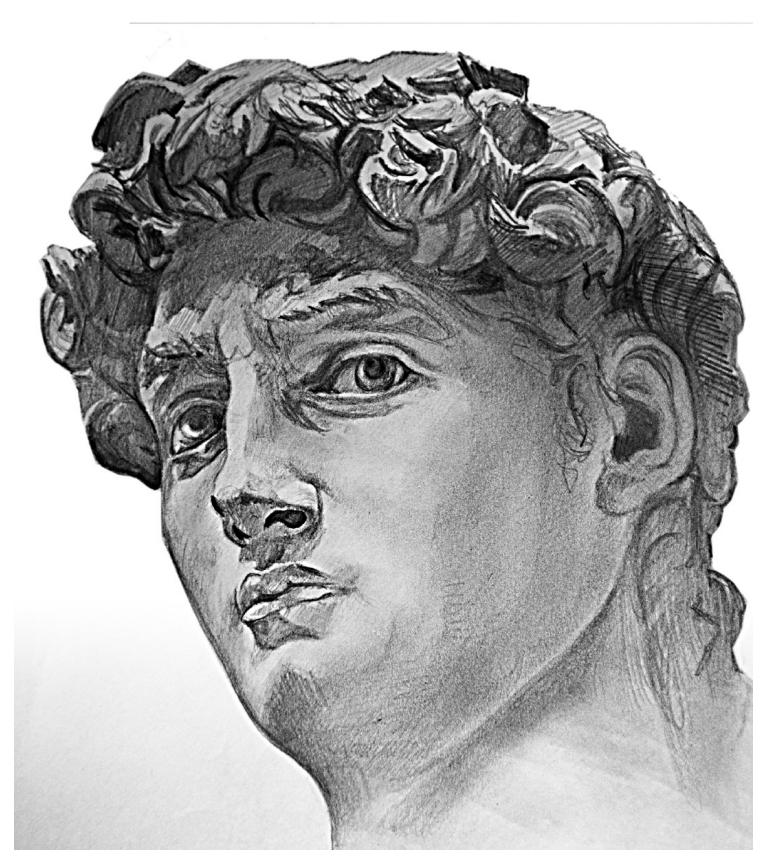

$\triangle$ Fig. 17 : dessin de l'auteur représentant la tête du fameux « David » de Michel-Ange de trois-quart face. 


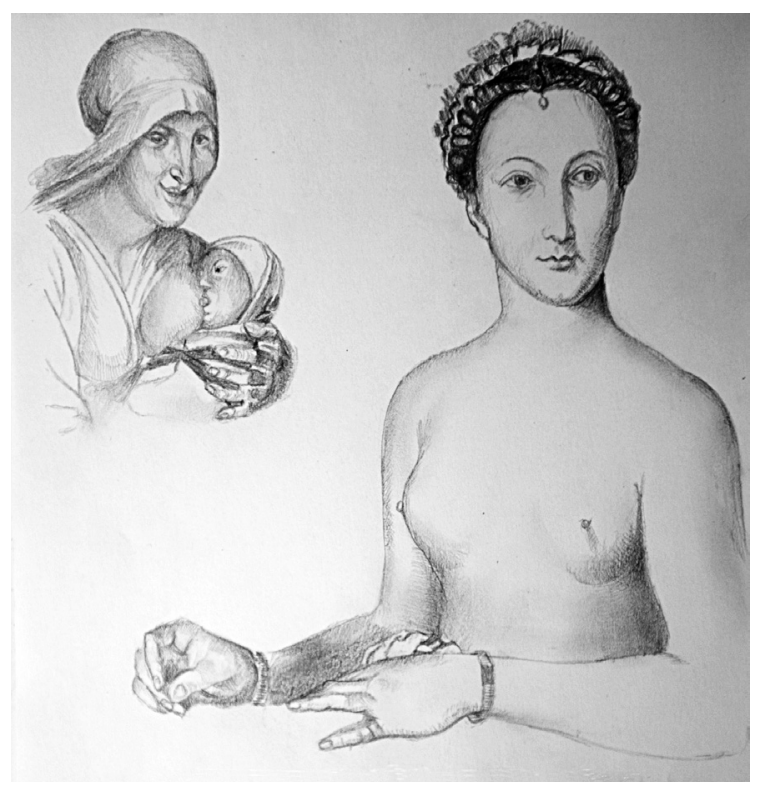

$\triangle$ Fig. 18 : dessin de l'auteur représentant Gabrielle d'Estrées peinte par François Clouet circa 1570. Ce visage féminin n’obéit pas aux proportions de Ricketts : le nez est exagérément allongé dans le sens vertical.

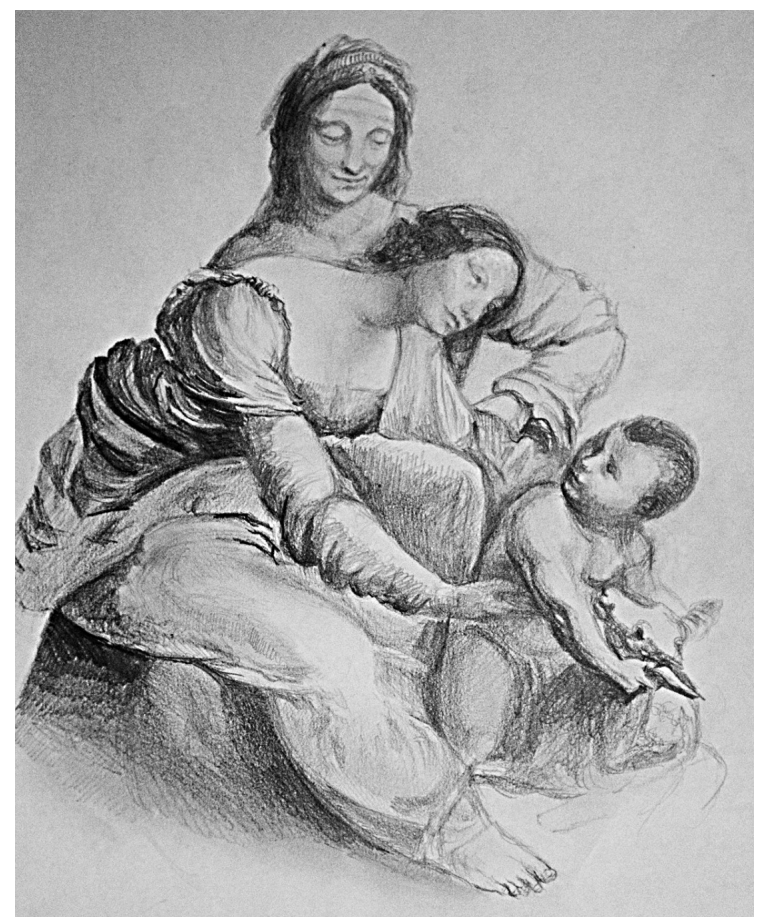

$\triangle$ Fig. 19 : dessin de l'auteur représentant « Sainte Anne, la vierge et l'enfant Jésus » d'après la toile du Louvres de Léonard de Vinci ou les 3 âges de la vie.

les proportions harmonieuses du visage de la beauté féminine : la mesure de la distance base du nez- ligne bicommissurale se situerait dans un rapport 1 tandis que la mesure de la distance ligne bicommissurale ligne du bord inférieur symphysaire vaudrait 1,618. La règle de Willis impliquerait alors que cette dernière distance est égale à la distance entre la ligne bipupillaire et la base du nez (fig. 12 proche de ce nombre dor).

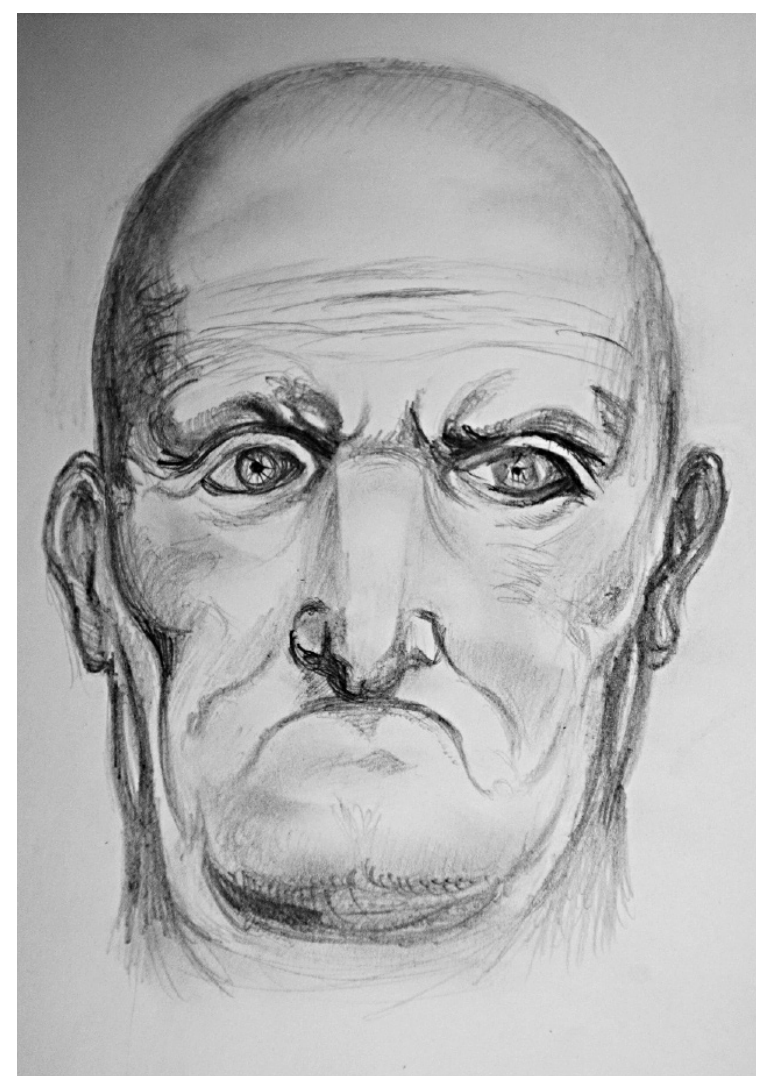

$\triangle$ Fig. 20 : dessin de l'auteur inspiré par une tête de vieillard de Léonard de Vinci.

\section{L'enfant et le vieillard}

Le détail de l'enfant Jésus dans "Sainte Anne, la vierge et l'enfant Jésus » de Léonard de Vinci montre le visage de l'enfant caractéristique avec une tête toute ronde, joufflue et un nez en trompette qui contraste avec le visage ovalaire typique de la morphologie adulte. La différence la plus marquante est la hauteur des yeux dans le visage de face. Ces derniers sont situés sur la ligne médiane au lieu de se situer aux deux-tiers chez l'adulte.

Dans l'étude de vieillard de Léonard de Vinci (fig. 20), on peut voir un visage ovalaire avec des rides expressives comme figées à demeure :

ride du pyramidal à la racine du nez;

$\checkmark$ rides frontales;

$\checkmark$ rides du lion;

I sillon nasogénien ;

patte d'oie de l'orbiculaire des paupières ;

$\checkmark$ effondrement des angles oraux avec perte des structures dentaires, du soutien de la lèvre et de la dimension verticale de l'étage inférieur de la face qui se traduit visuellement par une disposition des yeux sur la ligne médiane, comme chez l’enfant ;

$\checkmark$ effondrement de la pointe du nez (comme décrite par Christian Vacher in 10). 


\section{Léonard de Vinci et les trois âges de la vie}

La gradation des traits du visage en fonction de lâge des personnages constitue le thème du tableau du Louvres : La Vierge, Sainte Anne et l'enfant Jésus dont une ébauche est représentée plus haut. On est en droit de se demander si le sujet religieux ne constituait pas pour Léonard un prétexte pour étudier cette fascinante évolution dans le temps du visage humain. Ainsi, cette toile illustre magnifiquement que :

I De haut en bas, on part des formes les plus anguleuses aux formes les plus rondes.

I La rondeur d'un visage est donc bien souvent fonction de l'âge du sujet.

\section{CONCLUSION}

En pratique, ces considérations anatomiques ne sont que des bases nécessaires pour :

I savoir échapper aux canons, aux modes du temps, pour s'approcher au plus près des caractéristiques morphologiques de chaque individu ;

$\checkmark$ réaliser à la fois une œuvre utile et esthétique : être un artisan plutôt qu'un artiste ;

$\checkmark$ connaitre le Principe de Richardson : " il est impossible de voir ce que sont les choses à moins de savoir ce qu'elles doivent être ", que l'on peut contracter par « on ne voit réellement que ce que l'on comprend ».

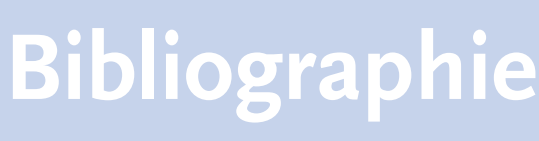

[1] Waller BM, et al. Fuglevand intramuscular electrical stimulation of facial muscles in humans and chimpanzees: Duchenne revisited and extended. Emotion 2006;6(3):367-382.

[2] Charrier J-L, Gaudy J-F, Bilweis C, Cannas B. Anatomie clinique. Éditions $C d P, 2007,224$ p.

[3] Dichamp J, Guilbert F, Vaillant J-M. Prothèse plastique faciale. Encycl Med Chir, Elsevier SAS Paris, Stomatologie, 22-087-M10, 7-1990;10 p.

[4] Gaudy J-F, Vacher C. Atlas d'anatomie clinique et chirurgicale des tissus superficiels de la tête et du cou. Éditions Elsevier-Masson, 2010, 288 p.
[5] Maillot C, Kahn J-L. Tête et Cou. Anatomie topographique. Paris : Éditions Springer-Verlag France, 2004, 222 p.

[6] Moreau A. Anatomie artistique - Précis d'anatomie osseuse et musculaire. Paris : Maloine, 1947. Réédition : Anatomie artistique de l'homme, 1975.

[7] Oberlin C, Vacher C, Berthelot JL. Précis d'Anatomie. Tome 1 - Ostéologie du thorax et du bassin, Anatomie de la tête et du cou. $11^{e}$ édition. Éditions Lavoisier, 2004.

[8] Richer P. Introduction à létude de la figure humaine. Gaultier, Magnier et Cie, 1902, 198 p.

[9] Rouvière H, Delmas A, Delmas V. Anatomie humaine : descriptive, topographique et fonctionnelle. Tête et cou. Elsevier Masson, $2002,654 \mathrm{p}$.
[10] Vacher C. Anatomie du vieillissement cranio-facial. EMC dentisterie-1 Elsevier-SAS editions 2004; 201-213.

[11] Vacher C, Accioli J, Lezy J-P. Surgical anatomy of the nose in the elderly: value of conservative rhinoplasty by the transoral route. Surg Radiol Anat 2002;24(3-4) :140-146.

[12] Van Volen L. Prothèse plastiques complexes et leurs problèmes esthétiques. Revue Française de Prothèse Maxillo-Faciale 1975;4(4):219-224.

[13] Vegter F, Hage JJ. Clinical anthropology and canons of the face in historical perspective. Plast Reconstr Surg 2000;106(5) :1090-1096. 